\title{
Anaplastic thyroid carcinoma diagnosed after treatment of lenvatinib for papillary thyroid carcinoma
}

\author{
Haruhiko Yamazaki', Hiroyuki Iwasaki', Nobuyasu Suganuma1, Soji Toda1, \\ Katsuhiko Masudo², Hirotaka Nakayama ${ }^{3}$, Yasushi Rino ${ }^{3}$ and Munetaka Masuda ${ }^{3}$ \\ 'Department of Breast and Endocrine Surgery, Kanagawa Cancer Center, Yokohama City, Kanagawa, Japan, \\ 2Department of Breast and Thyroid Surgery, Yokohama City University Medical Center, Yokohama City, Kanagawa, \\ Japan, and 'Department of Surgery, Yokohama City University School of Medicine, Yokohama City, Kanagawa, Japan
}

Correspondence

should be addressed

to H Yamazaki

Email

paruo0413@gmail.com

\section{Summary}

Anaplastic transformation of a primary thyroid tumor whose process can be followed is rare. The objective this study is to report a case of anaplastic transformation of locally advanced papillary thyroid carcinoma after treatment with lenvatinib. A 74-year-old woman consulted a local physician because of cough and bloody sputum. Thyroid cancer with tracheal invasion was suspected on computed tomography (CT) imaging, and she visited our hospital for treatment. We suspected anaplastic thyroid cancer (ATC) and core needle biopsy was performed. Histologic sections of the core needle biopsy showed that the tumor formed a papillary structure, and we diagnosed papillary thyroid carcinoma. Surgery would have been difficult, and we initiated lenvatinib at a low dose of $8 \mathrm{mg} /$ day. CT on day 40 of lenvatinib treatment revealed that the thyroid tumor had shrunk remarkably. CT on day 111 revealed that tumor regrowth and tracheal invasion had been exacerbated. Core needle biopsy was performed, and histologic sections of the core needle biopsy that was performed after regrowth of the tumor showed that individual cancer cells had large, irregular nuclei, and necrosis was also observed. The immunohistochemical findings were negative for thyroglobulin, and only a few cells were positive for thyroid transcription factor 1, and we diagnosed ATC. Anaplastic transformation of the target lesion may be one of the causes of lenvatinib treatment failure in differentiated thyroid carcinoma.

\section{Learning points:}

- Anaplastic transformation of a primary thyroid tumor whose process can be followed is rare.

- The resistance mechanism of lenvatinib in treatment for differentiated thyroid carcinoma has not been clarified.

- Anaplastic transformation of the target lesion may be one of the causes of lenvatinib treatment failure in differentiated thyroid carcinoma.

\section{Background}

Anaplastic thyroid carcinoma (ATC), although it accounts for only $2 \%$ of all thyroid cancers, is one of the most lethal human neoplasms, with a 1-year survival rate of only $18 \%$ (1). It is generally accepted that ATC either occurs de novo or progresses from a pre-existing, welldifferentiated thyroid carcinoma (WDTC) through a multistep process of genetic and epigenetic changes that lead to clonal expansion and neoplastic development (2). Cases of development of anaplastic transformation after lymph node recurrence have been reported (3). However, anaplastic transformation of a primary thyroid tumor whose process can be followed is rare. We report a case 
of locally advanced papillary thyroid carcinoma that was transformed into ATC after treatment with lenvatinib.

\section{Case presentation}

A 74-year-old woman with a history of treatment for cerebral infarction consulted a local physician because of cough and bloody sputum. Thyroid cancer with tracheal invasion was suspected on computed tomography (CT) imaging, and she visited our hospital for treatment.

\section{Investigation}

At the time of her visit, we palpated a tumor measuring $3 \mathrm{~cm}$ in the right lobe of the thyroid gland. Her blood test results showed thyroid-stimulating hormone of $3.02 \mu \mathrm{IU} / \mathrm{mL}$, free triiodothyronine of $2.24 \mathrm{pg} / \mathrm{mL}$, free thyroxine of $1.08 \mathrm{ng} / \mathrm{mL}$, thyroglobulin of $153 \mathrm{ng} / \mathrm{mL}$, and anti-thyroglobulin antibody of less than $10 \mathrm{IU} / \mathrm{mL}$. Ultrasonography revealed a cystic mass in the right lobe of the thyroid, a low-echoic area with unclear borders that was developing in the mediastinum, and a metastatic lymph node (Fig. 1A and B). CT revealed a thyroid tumor protruding into the tracheal lumen (Fig. 1C and D). There were no distant metastases. Bronchoscopic examination revealed a tumor, which bled easily, that projected into the lumen from the right side and the membranous part of the trachea. We suspected ATC and core needle biopsy was performed on the low-echoic area. Histologic sections of the core needle biopsy showed that the tumor formed a papillary structure (Fig. 1E and F), and we diagnosed papillary thyroid carcinoma (PTC).

\section{Treatment}

The patient could not receive general anesthesia because the tumor projected into the lumen of the trachea whose minimum diameter was $4 \mathrm{~mm}$ and was bleeding, making intubation impossible. Furthermore, tracheal invasion area was too wide to do reconstruction or tracheostomy. Surgery would have been difficult, and we discussed possible treatments with an oncologist and a radiologist. External irradiation and best supportive care were considered as therapeutic options. However, lenvatinib was judged likely to be more effective than these options. The efficacy and safety of lenvatinib for treatment of conditions such as this case had not been established. We fully informed the patient and her family of the benefits and risks of each treatment method before receiving their consent for lenvatinib treatment. Because there were

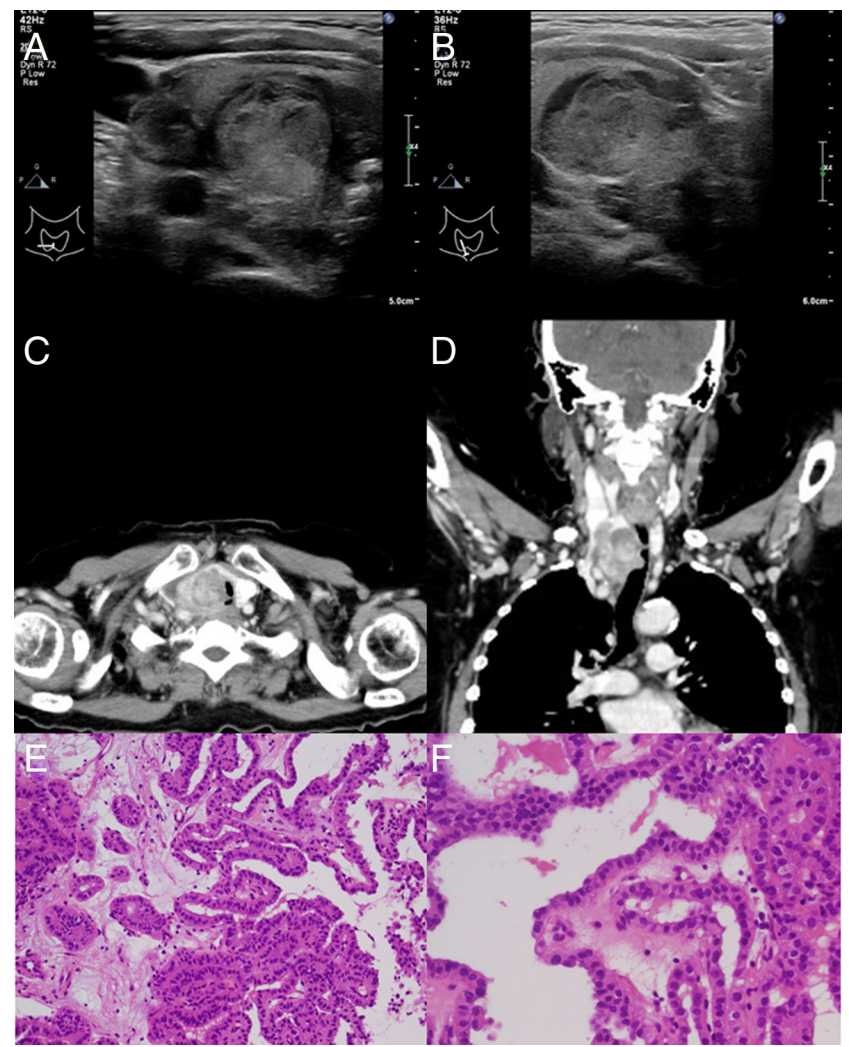

\section{Figure 1}

Images and pathological examinations of initial visit. (A and B) Ultrasonography revealed a cystic mass in the right lobe of the thyroid, a low-echoic area with unclear borders that was developing in the mediastinum and a metastatic lymph node. ( $C$ and D) Computed tomography $(\mathrm{CT})$ revealed a thyroid tumor protruding into the tracheal lumen. ( $E$ and F) Histologic sections of the core needle biopsy showed that the tumor formed a papillary structure. Individual cancer cells had nuclear grooves, and findings suggestive of nuclear inclusions were also observed. E: Original magnification $\times 200$; F: Original magnification $\times 400$.

concerns about promoting bleeding and the formation of a fistula between the tumor and the trachea, we initiated lenvatinib at a low dose of $8 \mathrm{mg} /$ day.

\section{Outcome and follow-up}

CT on day 40 of lenvatinib treatment revealed that the thyroid tumor had shrunk remarkably and that airway narrowing had improved (Fig. 2A and B), and CT on day 78 showed stable disease (Fig. 2C and D). No adverse events occurred, and interruption or reduction of lenvatinib was not required. However, CT on day 111 revealed that tumor regrowth and tracheal invasion had been exacerbated (Fig. 3C and D). The lenvatinib was increased to $14 \mathrm{mg} /$ day, and reduced to $10 \mathrm{mg} /$ day 5 days later because of grade 3 proteinuria. Although we increased the dose, the disease progression could not be under control. Ultrasonography 


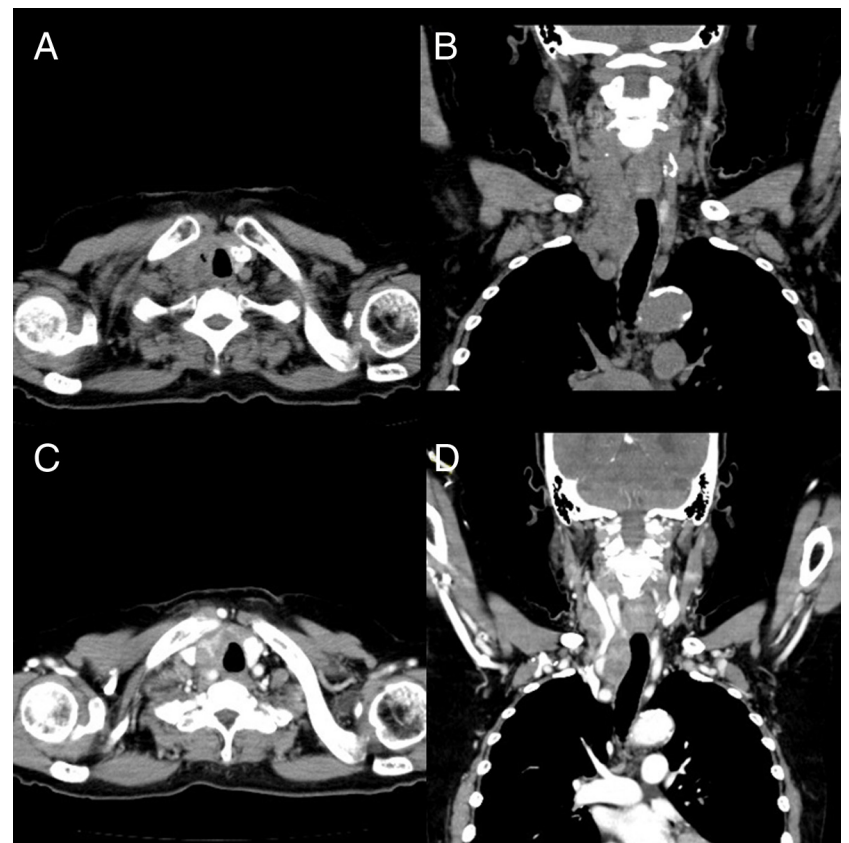

Figure 2

Images after lenvatinib treatment. (A and B) Computed tomography (CT) on day 40 of lenvatinib treatment revealed that the thyroid tumor had shrunk remarkably and airway narrowing had improved. (C and D) CT on day 78 of lenvatinib treatment showed stable disease.

revealed a tumor with a heterogeneous internal echo and an irregular border in the right lobe (Fig. $3 \mathrm{~A}$ and $\mathrm{B}$ ). The possibility that the biology of the tumor had changed was considered by images, and lenvatinib treatment failed because of progression of the disease. Core needle biopsy was performed again on the tumor in the right lobe to investigate microsatellite instability (MSI). Histologic sections of the core needle biopsy that was performed after regrowth of the tumor showed that individual cancer cells had large, irregular nuclei, and necrosis was also observed (Fig. 3E and F). The immunohistochemical findings were negative for thyroglobulin (Fig. 3G), and only a few cells were positive for thyroid transcription factor 1 (Fig. 3H), and we diagnosed ATC. MSI testing was negative, and we considered that lenvatinib was ineffective because the tumor had regrowth during the treatment. The patient stopped lenvatinib and chose BSC, and she died 6 days after interruption of lenvatinib.

\section{Discussion}

PTC accounts for the majority of thyroid carcinomas. The prognosis of PTC is good, with a disease-specific survival rate greater than $90 \%$ (4). However, the prognosis remains poor for patients with unresectable, advanced,

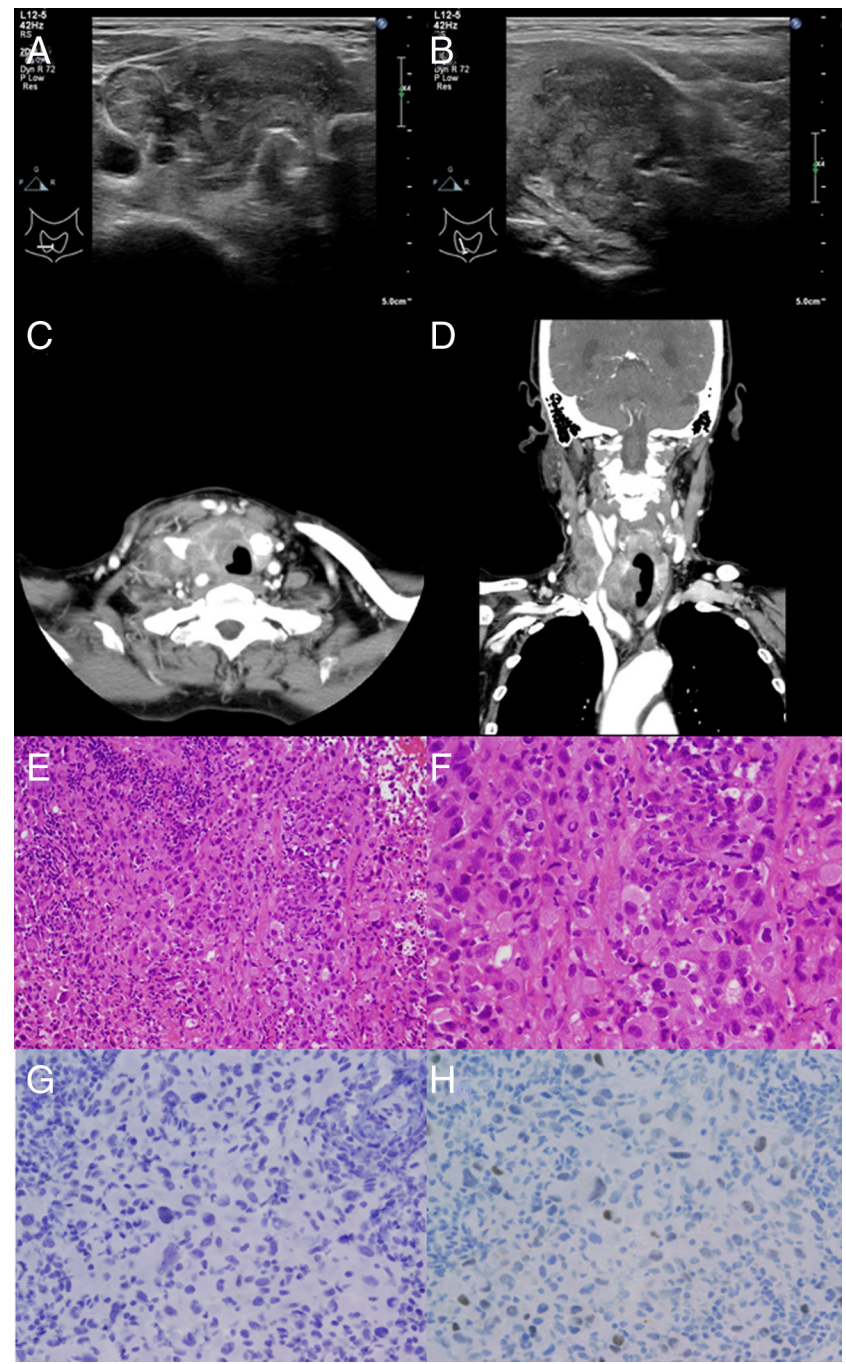

\section{Figure 3}

Images and pathological examinations after anaplastic transformation. (A and B) Ultrasonography revealed a tumor with a heterogeneous internal echo and an irregular border in the right lobe. (C and D) Computed tomography (CT) revealed a tumor with an irregular border in the right lobe, and tracheal invasion was exacerbated. (E and F) Histologic sections of the core needle biopsy that was performed after regrowth of the tumor showed that individual cancer cells had large, irregular nuclei, and necrosis was also observed. (E) Original magnification $\times 200$. (F) Original magnification $\times 400$. $(G$ and $H$ ) The immunohistochemical findings were negative for thyroglobulin (G), and only a few cells were positive for thyroid transcription factor $1(\mathrm{H})$. Original magnification $\times 400$.

or refractory PTC, with a median 10-year survival rate of $40-42 \%$; thus, it is important to develop effective treatment approaches for these patients (5). Surgery is the first choice for the initial treatment of PTC. However, it would have been difficult to perform surgery in this case. After consultation with the patient and her family, we started lenvatinib.

Lenvatinib, a multi-tyrosine kinase inhibitor, inhibits vascular endothelial growth factor receptors 1, 2, and 3; 
fibroblast growth factor receptors 1, 2, 3, and 4; plateletderived growth factor receptor $\alpha$, rearranged during transfection; and proto-oncogene receptor tyrosine kinase. In vitro studies have shown antineoplastic activity of lenvatinib in differentiated thyroid cancer, mainly because of its antiangiogenetic effects (6). After in vivo phase 1 and phase 2 study, the global phase 3 Study of (E7080) Lenvatinib in Differentiated Cancer of the Thyroid (SELECT) was conducted, and the lenvatinib showed a significant antitumor effect, with a median progressionfree survival (PFS) duration of 18.3 and 3.6 months in the lenvatinib and placebo groups, respectively (hazard ratio for progression or death: $0.21 ; 99 \%$ confidence interval (CI): 0.14-0.31; $P<0.001)$. The response rate, disease control rate, and clinical benefit rate were $64.7,87.7$, and $80.1 \%$, respectively (7).

The usual initial dose of lenvatinib is $24 \mathrm{mg} /$ day, and the dose is adjusted according to the occurrence of adverse events. However, we initiated lenvatinib at a low dose of $8 \mathrm{mg} /$ day because there were concerns that serious adverse events would occur (8). In SELECT trial, the initial dose of lenvatinib was $24 \mathrm{mg}$ /day. The dose was adjusted according to the occurrence of adverse events, and the minimum reduced dose was $8 \mathrm{mg} /$ day by protocol (7). So, we chose $8 \mathrm{mg} /$ day which was considered to be effective minimum dose as the initial dose in this case. CT on day 40 revealed that the primary thyroid tumor had shrunk remarkably by this reduced dose. This result indicated that there were some thyroid cancer patients for whom lenvatinib was efficacious at a low dose. Lenvatinib is used against radioactive iodine-refractory differentiated thyroid cancer (DTC). Therefore, the patients have already undergone total thyroidectomy. To our knowledge, this is the first case in which a primary thyroid tumor developed anaplastic transformation, as shown by histological examination, after lenvatinib treatment.

Two possible explanations are considered for the development of anaplastic changes in the primary thyroid tumor. One explanation is that the primary thyroid tumor had heterogeneity. Initially, the PTC component that showed sensitivity to lenvatinib shrank, but the ATC component that showed resistance to lenvatinib later increased. Sugitani et al. classified the clinical varieties of ATC into four types: the common type, the incidental type, anaplastic transformation at the neck lymph node(s), and anaplastic transformation at a distant site. PTC coexisted in $21 \%$ of the common type ATCs (1). In Japan, lenvatinib has also been used against ATC. Iwasaki et al. reported that the ATC patients exhibited a lenvatinib response rate of $17.4 \%$ and a disease control rate of $43.5 \%$, and the median OS time was 166 days (8). Lenvatinib is less effective against ATC than against PTC. PTC was diagnosed at the first visit by core needle biopsy, and lenvatinib was effective against the PTC components. However, there is a possibility that lenvatinib was not effective against the ATC components that coexisted with PTC in our patient. The other possible explanation is that anaplastic transformation of PTC was induced. The possibility that some gene mutation or mutations were induced by lenvatinib may be considered, because lenvatinib blocks a variety of signaling pathways. PTC and follicular tumors have been reported as sites of occurrence of ATC (1). According to the Vogelstein model, in many carcinomas, mutational inactivation of p53 is a crucial first step in the progression from adenoma to carcinoma (9). However, the majority of WDTCs have normal p53 genes, and in thyroid tumors, p53 gene inactivation, which is a late event in the carcinogenic process and occurs together with a marked increase in cell proliferation, seems to play an important role in the progression from differentiated to undifferentiated carcinoma (2). It was reported that when the same tumor contained WDTC and ATC components, the p53 mutation was restricted to the ATC components (9). ATCs acquire $\beta$-catenin and p53 mutations when arising de novo or dedifferentiating from WDTC. ATCs also contain other mutations, such as telomerase reverse transcriptase and BRAF mutations (10). Further research is needed on the mechanism of ATC occurrence. The results of our case may partly explain the cause of lenvatinib treatment failure in DTC patients.

We have reported the case of a PTC patient whose primary tumor developed anaplastic transformation after lenvatinib treatment. This result may indicate that anaplastic transformation of the target lesion is one of the causes of lenvatinib treatment failure in DTC.

\section{Declaration of interest}

The authors declare that there is no conflict of interest that could be perceived as prejudicing the impartiality of the research reported.

\section{Funding}

This research did not receive any specific grant from any funding agency in the public, commercial or not-for-profit sector.

\section{Patient consent}

Written informed consent was obtained from the patient for publication of this case report and accompanying images. 


\section{Author contribution statement}

$\mathrm{H} \mathrm{Y}$ prepared the manuscript. H I, N S, S T, K M and H N cared for the patient. $\mathrm{Y} R$ and $\mathrm{M} M$ comprehensively supervised this case report. All authors read and approved the final manuscript. The authors thank Enago (https://www.enago.jp/) for editing a draft of this manuscript.

\section{References}

1 Sugitani I, Miyauchi A, Sugino K, Okamoto T, Yoshida A \& Suzuki S. Prognostic factors and treatment outcomes for anaplastic thyroid carcinoma: ATC Research Consortium of Japan cohort study of 677 patients. World Journal of Surgery 201236 1247-1254. (https://doi. org/10.1007/s00268-012-1437-z)

2 Soares P, Lima J, Preto A, Castro P, Vinagre J, Celestino R, Couto JP, Prazeres H, Eloy C, Máximo V, et al. Genetic alterations in poorly differentiated and undifferentiated thyroid carcinomas. Current Genomics 201112 609-617. (https://doi. org/10.2174/138920211798120853)

3 Ito Y, Higashiyama T, Hirokawa M, Fukushima M, Inoue H, Yabuta T, Tomoda C, Uruno T, Kihara M, Takamura Y, et al. Prognosis of patients with papillary carcinoma showing anaplastic transformation in regional lymph nodes that were curatively resected. Endocrine Journal 200855 985-989. (https://doi.org/10.1507/endocrj.K08E148)

4 Matsuzu K, Sugino K, Masudo K, Nagahama M, Kitagawa W, Shibuya H, Ohkuwa K, Uruno T, Suzuki A, Magoshi S, et al. Thyroid lobectomy for papillary thyroid cancer: long-term follow-up study of 1088 cases. World Journal of Surgery 201438 68-79. (https://doi. org/10.1007/s00268-013-2224-1)

5 Cabanillas ME \& Habra MA. Lenvatinib: role in thyroid cancer and other solid tumors. Cancer Treatment Reviews 201642 47-55. (https:// doi.org/10.1016/j.ctrv.2015.11.003)

6 Ferrari SM, Ruffilli I, Centanni M, Virili C, Materazzi G, Alexopoulou M, Miccoli M, Antonelli A \& Fallahi P. Lenvatinib in the therapy of aggressive thyroid cancer: state of the art and new perspectives with patents recently applied. Recent Patents on AntiCancer Drug Discovery 201813 201-208. (https://doi.org/10.2174/157 4892813666180220110729)

7 Schlumberger M, Tahara M, Wirth LJ, Robinson B, Brose MS, Elisei R, Habra MA, Newbold K, Shah MH, Hoff AO, et al. Lenvatinib versus placebo in radioiodine-refractory thyroid cancer. New England Journal of Medicine 2015372 621-630. (https://doi.org/10.1056/ NEJMoa1406470)

8 Iwasaki H, Yamazaki H, Takasaki H, Suganuma N, Nakayama H, Toda S \& Masudo K. Lenvatinib as a novel treatment for anaplastic thyroid cancer: a retrospective study. Oncology Letters 201816 7271-7277. (https://doi.org/10.3892/ol.2018.9553)

9 Eloy C, Ferreira L, Salgado C, Soares P \& Sobrinho-Simões M. Poorly differentiated and undifferentiated thyroid carcinomas. Turk Patoloji Dergisi 201531 (Supplement 1) 48-59. (https://doi.org/10.5146/ tjpath.2015.01314)

10 Papp S \& Asa SL. When thyroid carcinoma goes bad: a morphological and molecular analysis. Head and Neck Pathology 20159 16-23. (https://doi.org/10.1007/s12105-015-0619-z)

Received in final form 18 August 2019

Accepted 27 August 2019 